\title{
Effects of Olmesartan and Azilsartan on Albuminuria and the Intrarenal Renin-Angiotensin System
}

\author{
Takeshi Takami, Sadanori Okada, Yoshihiko Saito, Yoko Nishijima, \\ Hiroyuki Kobori, Akira Nishiyama
}

\begin{abstract}
Purpose: Olmesartan and azilsartan decrease blood pressure more effectively than other angiotensin receptor blockers (ARBs). ARBs additionally decrease the urinary albumin to creatinine ratio (UACR), a urinary albumin marker, and urinary angiotensinogen (u-AGT), an intrarenal renin-angiotensin system activity marker. We examined the effects of these ARBs on blood pressure, UACR, and U-AGT in patients with uncontrolled hypertension.

Methods: Patients with uncontrolled hypertension treated with conventional ARBs, excluding olmesartan and azilsartan, for over 8 weeks were enrolled. We randomly switched patients from their prior ARBs to either olmesartan or azilsartan, and followed them for 24 weeks.
\end{abstract}

Results: Systolic blood pressure (SBP), diastolic blood pressure (DBP), and central systolic blood pressure (cSBP) significantly decreased at 24 weeks. UACR and u-AGT also decreased at 24 weeks in both groups. There were no significant differences in SBP, DBP, cSBP, UACR, or u-AGT between the groups. Therefore, we combined both groups for further analyses. After combining, SBP $(160.5 \pm 16.4$ to $139.6 \pm 15.6 \mathrm{~mm}$ $\mathrm{Hg}, \mathrm{P}<0.0001)$, DBP $(88.4 \pm 13.7$ to $80.7 \pm 13.2 \mathrm{~mm} \mathrm{Hg}, \mathrm{P}=$ $0.008), \operatorname{cSBP}(167.4 \pm 20.8$ to $146.6 \pm 24.6 \mathrm{~mm} \mathrm{Hg}, \mathrm{P}<0.0001)$, UACR (13.8 to $9.0 \mathrm{mg} / \mathrm{g}$ Cre, $P=0.0096)$, and $\mathrm{u}$-AGT (4.13 to $2.32 \mu \mathrm{g} / \mathrm{g}$ Cre, $P=0.0074)$ significantly decreased at 24 weeks. Patients with microalbuminuria (UACR $\geq 30 \mathrm{mg} / \mathrm{g}$ Cre) had significantly greater $\triangle \mathrm{UACR}(-39.4$ vs $0.27, \mathrm{P}=\mathbf{0 . 0 0 2 4})$ and $\Delta$ u-AGT $(-11.9$ vs $-\mathbf{0 . 6 1}, P=0.0235)$ than patients without microalbuminuria. The changes in $\mathrm{u}$-AGT were significantly associated with changes in UACR $(r=0.411, P=0.046)$; however, there was no significant relationship between the changes in u-AGT and those in SBP or DBP.

Conclusion: Olmesartan and azilsartan decreased blood pressure, UACR, and u-AGT more than the other ARBs, and exerted depressor and renoprotective effects.

Index Terms - blood pressure, urinary albumin, urinary angiotensinogen, angiotensin receptor blockers.

\section{INTRODUCTION}

A critical objective of antihypertensive therapy is not only the reduction in blood pressure, but also the protection of vital organs. The vital organs affected by hypertension

,Takeshi Takami, Department of Internal Medicine, Clinic Jingumae, Kashihara, Japan,

Sadanori Okada, Department of Cardiovascular Medicine, Nara medical University, Kashihara, Japan

Yoshihiko Saito, Professor of Department of Cardiovascular Medicine, Nara medical University, Kashihara, Japan

Yoko Nishijima, Department of CardioRenal and CerebroVascular Medicine, Faculty of Medicine, Kagawa University, Kagawa, Japan

Hiroyuki Kobori, Professor in the Departments of Pharmacology and of Nephrology, School of Medicine, International University of Health and Welfare, Narita, Japan

Akira Nishiyama, Professor of the Department of Pharmacology, Faculty of Medicine, Kagawa University, Kagawa, Japan include the heart, kidneys, and cerebral vessels, and injuries to these organs affect the prognosis of patients. In addition to their well-known depressor activities, angiotensin receptor blockers (ARBs) exhibit important cardiac and renal protective effects.

In recently published studies (i.e., the CANZONE [Comparison of Efficacy and Safety of Azilsartan and Olmesartan in Patients With Essential Hypertension] [1] and MUSCAT-4 [Multicenter Probe Study-4; Comparison of the Effects of Angiotensin II Type 1 Receptor Blockers] [2] studies), azilsartan and olmesartan, which have the most potent antihypertensive activities among the ARBs, were directly compared and analyzed. Although both azilsartan and olmesartan demonstrated potency, no differences were observed in study outcomes between these drugs.

Estimated glomerular filtration rate (eGRF) and urinary albumin are used as biomarkers of renal function. In particular, microalbuminuria is an important risk factor for cardiovascular events. ARBs are effective in reducing urinary albumin excretion, independently of their depressor effects [ 3 ], [ 4 ]. However, it has not been established whether the decreased urinary albumin is attributable to a class effect of the ARBs or a drug effect unique to individual ARBs. Investigations of the dependency of decreased urinary albumin on reduced blood pressure are important for studies of cardiovascular events. In the present study, the urinary albumin-to-creatinine ratio (UACR) was used as a marker of urinary albumin.

Classically, the renin-angiotensin system (RAS) is based on the concept of a circulating RAS, in which angiotensinogen, a substrate synthesized in the liver, is converted by the rate-limiting enzyme, renin, to angiotensin I, and then by angiotensin-converting enzyme (ACE) to angiotensin II (ATII); the latter is involved in control of blood pressure and fluid retention[ 5 ]. Moreover, tissue-specific local RASs have been attracting attention because of their potential organ-protecting effects that are independent of blood pressure control[ 5 ]. The importance of tissue-specific RASs in the kidneys[ 6 ] [ 7 ], brain[ 8 ], heart [9 ], [ 10], adrenal glands[ 11 ], and blood vessels [ 12 ] has been reported. Among these organ systems, the nephrons and tubulointerstitium in the kidneys contain all of the essential components for synthesis of ATII. Urinary angiotensinogen (u-AGT) is a specific marker of activation of the intrarenal RAS, reflecting activated intrarenal ATII, which is associated with deterioration of renal function due to chronic renal disease (CKD) [ 13 ]. As a biomarker of activated intrarenal RAS, u-AGT has been examined in patients with 
hypertension [ 14 ]- [ 16 ].

Recently, it was reported that olmesartan reduces urinary albumin and u-AGT [ 17 ]. We hypothesized that decreased $\mathrm{u}$-AGT contributes to the renal protective actions of ARBs through the inhibition of intrarenal RAS. Thus, in the present study, the effects of azilsartan and olmesartan on systolic blood pressure (SBP), diastolic blood pressure (DBP), central systolic blood pressure (cSBP), UACR, and u-AGT were assessed to evaluate the depressor and renal protective actions (anti-albuminuric and anti-intrarenal RAS effects) of both drugs.

\section{METHODS}

A.Study design:

This is a pilot study and a subanalysis of the Cardio study, where the effectiveness of azilsartan and olmesartan in controlling blood pressure (BP) and protecting the kidneys was evaluated. Outpatients with hypertension currently receiving therapy were recruited at the Department of Internal Medicine at Clinic Jingumae (Kashihara, Japan) between November 2012 and April 2014.

In the Cardio study, 24 patients with hypertension who were treated with ARBs, except azilsartan and olmesartan, but had uncontrolled BP, were enrolled. Patients with uncontrolled BP were defined according to the Japanese Society of Hypertension Guideline 2009 for the Management of Hypertension. They were prospectively and randomly switched from their current ARB to azilsartan ( $20 \mathrm{mg} /$ day) or olmesartan $(20 \mathrm{mg} /$ day) and were followed for 24 weeks . At baseline and after 24 weeks, SBP, DBP, heart rate (HR), cSBP, Augmentation Index, brachial-ankle pulse wave velocity, left ventricular mass index, left ventricular diastolic function, left ventricular systolic function, urinary albumin, u-AGT, eGFR, plasma ATII, and plasma aldosterone were determined.

We excluded patients with secondary hypertension, acute coronary syndrome, stroke, or malignant neoplasm within the previous 6 months, as well as those with serious renal disease, serious liver disease, pregnancy, or a history of allergy to azilsartan or olmesartan. The protocol of this study (Cardio study: unpublished) was approved by the ethics committee of Kagawa University Hospital and registered under University Hospital Medical Information Network ID UMIN000009847. All subjects provided written informed consent to participate.

B. Evaluation of clinical parameters:

We measured office BP, HR, c-SBP, UACR, u-AGT, plasma ATII, and plasma aldosterone (PAC) at baseline and after 24 weeks. Office BP and HR were determined as the mean of two measurements obtained in an office setting by the cuff method after at least 5 min of rest. cSBP was determined using the HEM-9000AI (Omron Healthcare, Kyoto, Japan), which is known to generate comparable values to those determined by generalized aorta-radial transfer function [18]. We measured u-AGT using a method described by Nishijima et al [19].

C.Statistical analysis:

The data were analyzed using JMP 12.2 (SAS Institute, Cary, NC). Paired t-tests were used to compare parametric data, while the Wilcoxon signed-rank test was used to compare nonparametric data before and after treatment. The Student's t-test or Wilcoxon rank-sum test was used for between-group comparisons. Linear regression analysis was performed to examine the correlation of $\Delta \mathrm{u}$-AGT/creatinine (Cre) with $\triangle \mathrm{UACR}, \triangle \mathrm{SBP}$, and $\triangle \mathrm{DBP}$, as well as $\triangle \mathrm{UACR}$ with $\triangle \mathrm{eGFR}$. A P-value of $<0.05$ was considered to represent statistical significance.

\section{RESULTS}

Table 1 summarizes the baseline characteristics of the patients. The treatment ARBs prior to switching medications were candesartan (11 cases), telmisartan (10 cases), valsartan ( 2 cases), and losartan (1 case). All ARBs were used at conventional doses. In addition to ARBs (and including overlap), the other prescribed hypotensive agents were calcium channel blockers (CCBs, 17 cases), diuretics (4 cases), $\beta$ blockers ( 1 case), and $\alpha$ blockers ( 1 case). We did not change these non-ARB medications throughout the study period.

Table1. Baseline characteristics, Values are mean, S.D. or $\%$ or median, IQR (Q1-Q3), N=24.

\begin{tabular}{lcc}
\hline Items & mean & S.D \\
\hline Age(years) & 65.79 & 10.74 \\
Sex $(\mathrm{male} \%)$ & 29.2 & \\
BMI $\left(\mathrm{kg} / \mathrm{m}^{2}\right)$ & 24.62 & 3.08 \\
$\mathrm{SBP}(\mathrm{mmHg})$ & 160.5 & 16.4 \\
$\mathrm{DBP}(\mathrm{mmHg})$ & 88.4 & 13.7 \\
$\mathrm{HR}(\mathrm{bpm})$ & 77.3 & 12.5 \\
$\mathrm{cSBP}(\mathrm{mmHg})$ & 167.4 & 20.8 \\
$\mathrm{LDLC}(\mathrm{mg} / \mathrm{dL})$ & 134.7 & 22.1 \\
$\mathrm{TG}(\mathrm{mg} / \mathrm{dL})$ & 161.0 & 80.2 \\
$\mathrm{hsCRP}(\mathrm{mg} / \mathrm{dL})$ & 0.096 & 0.099 \\
$\mathrm{BS}(\mathrm{mg} / \mathrm{dL})$ & 102.4 & 22.9 \\
$\mathrm{Hb}(\mathrm{g} / \mathrm{dL})$ & 14.3 & 1.4 \\
$\mathrm{HbA} 1 \mathrm{c}(\%)$ & 5.98 & 1.08 \\
$\mathrm{eGFR}\left(\mathrm{ml} / \mathrm{min} / 1.7 \mathrm{~m}^{2}\right)$ & 72.5 & 17.6 \\
$\mathrm{AT} \mathrm{II}(\mathrm{pg} / \mathrm{mL})$ & 12.0 & 6.4 \\
PAC $(\mathrm{pg} / \mathrm{mL})$ & 140.2 & 46.0 \\
\hline & Median & IQR \\
& & $(\mathrm{Q} 1-\mathrm{Q} 3)$ \\
\hline $\mathrm{UACR}(\mathrm{mg} / \mathrm{g} . \mathrm{Cre})$ & 13.8 & $4.8-45.0$ \\
$\mathrm{u}-\mathrm{AGT}(\mu \mathrm{g} / \mathrm{g}$ Cre $)$ & 4.13 & $0.76-12.73$ \\
\hline
\end{tabular}

Abbreviations: S.D.; standard deviation, IQR; interquartile range, BMI; body mass index, SBP; systolic blood pressure

DBP; diastolic blood pressure, HR; heart rate, bpm; beats per minute, cSBP; central systolic blood pressure, LDLC; low density lipoprotein cholesterol, TG; triglyceride, hs-CRP; high-sensitivity C-reactive protein, BS; fasting blood glucose, Hb; hemoglobin, HbAlc; hemoglobin Alc, eGFR; estimated glomerular filtration rate, AT II; plasma angiotensin II concentration, PAC; plasma aldosterone concentration, UACR; urinary albumin to creatinine ratio, $u$-AGT; urinary angiotensinogen

In the Cardio study, the following parameters were analyzed: brachial SBP (olmesartan: $157 \pm 5$ to $137 \pm 12 \mathrm{~mm}$ $\mathrm{Hg}, \mathrm{P}=0.001$ vs azilsartan: $164 \pm 5$ to $142 \pm 18 \mathrm{~mm} \mathrm{Hg}, \mathrm{P}=$ 0.004), cSBP (olmesartan: $159 \pm 13$ to $139 \pm 18 \mathrm{~mm} \mathrm{Hg}, \mathrm{P}=$ 0.0009 vs azilsartan: $176 \pm 24$ to $154 \pm 29 \mathrm{~mm} \mathrm{Hg}, \mathrm{P}=0.011$ ), PAC (olmesartan: $146.6 \pm 51.2$ to $109.0 \pm 43.9 \mathrm{pg} / \mathrm{mL}, \mathrm{P}=$ 
0.0007 vs azilsartan: $133.9 \pm 41.5$ to $92.4 \pm 49.5 \mathrm{pg} / \mathrm{mL}, \mathrm{P}=$ 0.002), UACR (olmesartan: 12.5 [7.4-64.0] to 9.7 [4.5-22.9] $\mathrm{mg} / \mathrm{gCre}, \mathrm{P}=0.052$ vs azilsartan: $17.2[3.8-42.7]$ to 7.6 [4.5-17.4] mg/g Cre, $\mathrm{P}=0.17$ ), and u-AGT (olmesartan: 4.1 [0.76-8.5] to 2.5 [0.15-5.6] $\mu \mathrm{g} / \mathrm{g}$ Cre, $\mathrm{p}=0.11$ vs azilsartan: $5.9[0.99-17.3]$ to $2.1[0.42-3.7] \mu \mathrm{g} / \mathrm{g}$ Cre, $\mathrm{P}=0.04)$. In the above data, SBP, cSBP, and PAC are expressed as means \pm S.D., while UACR and u-AGT are expressed as median [interquartile range (IQR)]. The baselines in both treatment groups were similar, except for cSBP. The other measured parameters showed no change from baseline in both groups. However, there were no significant differences between the ARB groups in the above parameters; accordingly, we analyzed the changes in BP, cSBP, UACR, and u-AGT in both groups combined.

Table 2. Changes in parameters over the 24-week study (t-tests)

\begin{tabular}{lccc}
\hline & Baseline & Week 24 & \\
\hline & Mean(S.D.) & Mean (S.D.) & Pvalue \\
\hline SBP $(\mathrm{mm} \mathrm{Hg})$ & $160.5(16.4)$ & $139.6(15.6)$ & $<.0001$ \\
DBP(mm Hg) & $88.4(13.7)$ & $80.7(13.2)$ & 0.008 \\
cSBP (mm Hg) & $167.4(20.8)$ & $146.6(24.6)$ & $<.0001$ \\
HR (bpm) & $77.3(12.5)$ & $72.7(12.3)$ & 0.0112 \\
eGFR & & & \\
(mL/min/1.73 m $)$ & $72.5(17.6)$ & $69.3(17.9)$ & 0.07 \\
HbA1c $(\%)$ & $5.98(1.08)$ & $6.00(1.05)$ & 0.3892 \\
LDLC $(\mathrm{mg} / \mathrm{dL})$ & $134.7(22.1)$ & $121.5(23.8)$ & 0.008 \\
ATII (pg/mL) & $12.0(6.4)$ & $27.1(50.6)$ & 0.159 \\
PAC (pg/mL) & $140.2(46.0)$ & $100.7(46.5)$ & $<.0001$ \\
\hline
\end{tabular}

In the combined parametric data, SBP, DBP , cSBP, PAC, $\mathrm{HR}$, and LDL-cholesterol significantly decreased at 24 weeks compared to baseline values (Table 2). In addition, UACR and $\mathrm{u}$-AGT significantly decreased at 24 weeks compared to baseline values (Table3).

Table3. Changes in urinary albumin and urinary angiotensinogen over the 24-week study (Wilcoxon test)

\begin{tabular}{|c|c|c|c|c|}
\hline & Baseline & \multicolumn{2}{|c|}{ Week 24} & \\
\hline & $\begin{array}{ll}\text { Median } & \text { IQR } \\
& (\mathrm{Q} 1-\mathrm{Q} 3)\end{array}$ & Median & $\begin{array}{l}\text { IQR } \\
\text { (Q1-Q3 }\end{array}$ & $\begin{array}{l}\text { P value } \\
\text { 3) }\end{array}$ \\
\hline $\begin{array}{c}\text { UACR } \\
\text { (mg/g Cre) } \\
\text { u-AGT }\end{array}$ & $13.8(4.8-45.0)$ & $9.0(4.5-$ & 17.6) & 0.0096 \\
\hline$(\mu \mathrm{g} / \mathrm{g}$ Cre $)$ & $4.13(0.76-12.73)$ & $2.32(0.2$ & $5-4.48)$ & 0.0074 \\
\hline
\end{tabular}

The changes in UACR and u-AGT were significantly greater in the microalbuminuria group (UACR $\geq 30 \mathrm{mg} / \mathrm{g}$ Cre) than in the non-microalbuminuria group (UACR $<30 \mathrm{mg} / \mathrm{g} \mathrm{Cre}$ ) (Table4).

\begin{tabular}{lccc}
\hline \multicolumn{5}{c}{ Table 4. Changes in UACR and u-AGT } \\
\hline \multicolumn{5}{c}{$\begin{array}{c}\text { UACR }<30 \\
\mathrm{mg} / \mathrm{g} \text { Cre }(\mathrm{N}=16)\end{array}$} & $\begin{array}{c}\mathrm{UACR} \geqq 30 \\
\mathrm{mg} / \mathrm{g} \text { Cre }(\mathrm{N}=8)\end{array}$ & P value \\
\hline $\begin{array}{l}\text { Change in } \\
\text { UACR } \\
\text { Change in } \\
\text { u-AGT }\end{array}$ & $0.27(-3.2 / 1.7)$ & $-39.4(-52.3 /-15.4)$ & 0.0024 \\
\hline
\end{tabular}

The changes in u-AGT were significantly associated with changes in UACR. However, there was no significant relationship between the changes in $\mathrm{u}-\mathrm{AGT}$ and the changes in SBP or DBP (Table 5) There was also no significant relationship between the changes in UACR and the changes in eGFR $(\mathrm{r}=0.124, \mathrm{P}=0.571)$.

Table5.Correlation between changes in urinary angiotensinogen and changes in urinary albumin to creatinine ratio, systolic blood pressure and diastolic blood pressure

\begin{tabular}{lcc}
\hline & \multicolumn{3}{c}{ Changes in u-AGT $(\mu \mathrm{g} / \mathrm{g}$ Cre $)$} \\
& $\mathrm{r}$ & $\mathrm{P}$ value \\
\hline Changes in UACR $(\mathrm{mg} / \mathrm{g}$ Cre $)$ & 0.411 & 0.046 \\
Changes in SBP $(\mathrm{mmHg})$ & -0.133 & 0.536 \\
Changes in DBP $(\mathrm{mmHg})$ & 0.355 & 0.089 \\
\hline
\end{tabular}

\section{DISCUSSION}

In the Cardio (the Difference of the Effect of ARB on Blood Pressure and Cardiac Function in Patients with Essential Hypertension) study (unpublished), olmesartan (showing decreasing tendencies in both u-AGT and UACR) and azilsartan (showing a significant decrease in only u-AGT and a decreasing tendency in UACR) exhibited significantly greater depressor and renal protective effects compared with those of other ARBs. Moreover, cardiovascular markers (i.e., arterial stiffness, left ventricular mass index, and left ventricular diastolic function) remained unchanged in the olmesartan and azilsartan groups. There were no differences in the measured parameters between the groups. Thus, these groups were combined in the present subanalysis to assess the depressor and renal protective effects.

In the present subanalysis, the depressor and renal protective effects were expressed as differences between values before and after switching the ARBs to olmesartan or azilsartan. The antihypertensive effect observed in this subanalysis was comparable to that observed in the CANZONE study [ 1 ] and the MUSCAT-4 study [ 2 ]. Regarding the renal protective effects, urinary albumin and u-AGT both tended to decrease in the olmesartan group, as observed in the study by Mizushige et al[ 17 ]. These authors reported that urinary albumin and u-AGT simultaneously decreased after olmesartan was administered to patients with metabolic syndrome [ 17 ]. The present study, which included few hypertensive patients with metabolic syndrome (olmesartan group: body mass index, 24), showed a similar tendency.

As previous studies have compared only the depressor effect between olmesartan and azilsartan treatments[ 1 ], [ 2 ], the present study, to our knowledge, is the first to directly compare the renal protective effects of the two drugs. Azilsartan and olmesartan exhibited renal protective effects (based on significant reductions in $\mathrm{u}$-AGT and UACR compared to baseline values) that were independent of their depressor effects. However, no difference was observed in the renal protective effect between the two drug groups.

PAC significantly decreased in both drug groups. The patients were followed only for 24 weeks in the present study; therefore, a follow-up period of 1 year will be necessary in future studies investigating whether olmesartan and azilsartan are effective in protecting against aldosterone breakthrough. However, some studies have already reported that olmesartan is unlikely to cause aldosterone breakthrough [ 20 ], [ 21 ]. Study limitations:

The present study is a pilot study, and its sample size is small. Furthermore, because the present subanalysis was 
performed on a combined group of patients receiving azilsartan or olmesartan, further studies with a larger sample size are needed to investigate whether differences in renoprotective effects exist between these drugs.

\section{CONCLUSION}

Azilsartan and olmesartan demonstrated a potent depressor activity, relieved albuminuria, and improved u-AGT, a marker of intrarenal RAS. Changes in u-AGT positively correlated with those in UACR, but did not correlate with those in blood pressure. The drugs were shown to not only relieve albuminuria but also markedly improve the intrarenal RAS, particularly in patients with hypertension exhibiting microalbuminuria. The data suggest that azilsartan and olmesartan have potent anti-albuminuric and anti-intrarenal RAS activity as well as potent depressor activity. These clinical findings support the hypothesis, based on prior observations [ 22 ]- [ 25 ], that attenuation of albuminuria with ATII blockade is associated with reductions in intrarenal RAS activity.

\section{DISCLOSURE}

The author reports no conflicts of interest related to this work.

\section{REFERENCES}

[ 1 ] Shiga Y, Miura S, Motozato K, et al. Comparison of efficacy and safety of azilsartan and olmesartan in patients with essential hypertension a randomized and prospective study (CANONE Study). Int Heart J.2017;58(3):416-421.

[ 2 ] Kakio Y, Uchida HA, Umebayashi R, et al. Practical efficacy of olmesartan versus azilsartan in patients with hypertension: a multicenter randomized-controlled trial (MUSCAT-4 study). Blood Press Monit 2017 ;22(2):59-67.

[ 3 ] Brenner BM, Cooper ME, de Zeeuw D, et al. Effects of losartan on renal and cardiovascular outcomes in patients with type 2 diabetes and nephropathy. N Engl J Med. 2001;345(12):861-869.

[ 4 ] Lewis EJ, Hunsicker LG, Clarke WR, et al. Renoprotective effect of the angiotensin-receptor antagonist irbesartan in patients with nephropathy due to type 2 diabetes. N Engl J Med.2001;345(12):851-860.

[ 5 ] Dzau VJ, Re R. Tissue angiotensin system in cardiovascular medicine. A paradigm shift? Circulation1994;89(1):493-498.

[ 6 ] Kobori H, Nangaku M, Navar LG, Nishiyama A. The intrarenal renin-angiotensin system: from physiology to the pathobiology of hypertension and kidney disease. Pharmacol Rev.2007 ;59(3):251-287.

[ 7 ] Campbell DJ, Lawrence AC, Towrie A, Kladis A, Valentijn AJ. Differential regulation of angiotensin peptide levels in plasma and kidney of the rat. Hypertension 1991. ;18(6):763-773.

[ 8 ] Baltatu O, Silva JA, Ganten D. Bader M. The brain renin-angiotensin system modulates angiotensin II-induced hypertension and cardiac hypertrophy. Hypertension 2000. ;35(1):409-412.

[ 9] Dell'Italia LJ, Meng QC, Balcells E, et al. Compartmentalization of angiotensin II generation in the dog heart. Evidence for independent mechanisms in intravascular and interstitial spaces. J Clin Invest 1997. ;100(2):253-258.

[ 10 ] Rafiq K, Noma T, Fujisawa Y, et al. Renal sympathetic denervation suppresses de novo podocyte injury and albuminuria in rats with aortic regurgitation. Circulation 2012. ;125(11):1402-1413.

[ 11 ] Mazzocchi G, Malendowicz LK, Markowska A, Albertin G, Nussdorfer GG. Role of adrenal renin-angiotensin system in the control of aldosterone secretion in sodium-restricted rats. Am J Physiol Endocrinol Metab $2000 ; 278(6)$ :E1027-E1030.

[ 12 ] Griendling KK, Minieri CA, Ollerenshaw JD, Alexander RW. Angiotensin II stimulates NADH and NADPH oxidase activity in cultured vascular smooth muscle cells. Circ Res.1994;74(6):1141-1148.

[ 13 ] Yamamoto T, Nakagawa T, Suzuki H, et al. Urinary angiotensinogen as a marker of intrarenal angiotensin II activity associated with deterioration of renal function in patients with chronic kidney disease. J Am Soc Nephrol.2007 ;18(5):1558-1565

[ 14 ] Kobori H, Alper AB, Shenava R, et al. Urinary angiotensinogen as a novel biomarker of the intrarenal renin-angiotensin system status in hypertensive patients. Hypertension.2009;53(2):344-350.

[ 15 ] Kobori H, Urushihara M, Xu JH, Berenson GS, Navar LG. Urinary angiotensinogen is correlated with blood pressure in men (Bogalusa Heart Study). J Hypertens. 2010 ;28(7):1422-1428.

[ 16 ] Konishi Y, Nishiyama A, Morikawa T, et al. Relationship between urinary angiotensinogen and salt sensitive of blood pressure in patients with IgA nephropathy. Hypertension.2011;58(2):205-211.

[ 17 ] Mizushige $\mathrm{T}$, Kobori $\mathrm{H}$, Hitomi $\mathrm{H}$, et al. Urinary angiotensinogen could be a prognostic marker of the renoprotection of olmesartan in metabolic syndrome patients. Int J Mol Sci.2016;17(11):1800.

[ 18 ] Takazawa K, Kobayashi H, Shindo N, Tanaka N, Yamashina A. Relationship between radial and central arterial pulse wave and evaluation of central aortic pressure using the radial arterial pulse wave. Hypertens Res.2007 ;30(3):219-228.

[ 19] Nishijima Y, Kobori H, Kaifu K, et al. Circadian rhythm of plasma and urinary angiotensinogen in healthy volunteers and in patients with chronic kidney disease. J Renin Angiotensin Aldosterone Syst. $2014 ; 15(4): 505-508$.

[ 20 ] Ichikawa S, Takayama Y. Long-term effects of olmesartan, an Ang II receptor antagonist, on blood pressure and the renin-angiotensin-aldosterone system in hypertensive patients. Hypertens Res.2001;24(6):641-646.

[ 21 ] Sezai A, Soma M, Hata M, et al. Effects of olmesartan on the renin-angiotensin-aldosterone system for patients with essential hypertension after cardiac surgery-investigation using a candesartan change-over study. Ann Thorac Cardiovasc Surg 2011 ;25(5):487-493.

[ 22 ] Kamiyama M, Garner MK, Farragut KM, et al. Detailed localization of augmented angiotensinogen mRNA and protein in proximal tubule segments of diabetic kidneys in rats and humans. Int $\mathrm{J}$ Biol Sci.2014; $10(5): 530-542$.

[ 23 ] Kamiyama M, Urushihara M, Morikawa $T$, et al. Oxidative stress/angiotensinogen/renin-angiotensin system axis in patients with diabetic nephropathy. Int J Mol Sci 2013. ;14(11):23045-23062.

[ 24 ] Kobori H, Kamiyama M, Harrison-Bernard LM, Navar LG. Cardinal role of the intrarenal renin-angiotensin system in the pathogenesis of diabetic nephropathy. J Investig Med.2013;61(2):256-264.

[ 25 ] Sofue T, Kiyomoto H, Kobori H, et al. Early treatment with olmesartan prevents juxtamedullary glomerular podocyte injury and the onset of microalbuminuria in type 2 diabetic rats. Am J Hypertens.2012;25(5):604-611. 